\title{
Redes globales de producción y proveedores locales: los empresarios sonorenses frente a la expansión de la industria automotriz*
}

\author{
Álvaro Bracamonte Sierra \\ y Oscar F. Contreras**
}

Resumen. En el contexto de la severa crisis por la que atraviesan las grandes empresas automotrices estadounidenses, una de las estrategias adoptadas ha sido la re-localización de sus plantas armadoras en México. En este artículo se analiza el caso del complejo automotriz liderado por Ford en Hermosillo, Sonora, mostrando que este tipo de aglomeraciones comandadas por empresas transnacionales generan derramas tecnológicas y propician la acumulación de capacidades locales. En particular, se explora el proceso de creación de pequeñas y medianas empresas locales vinculadas con las redes globales de la industria automotriz.

Palabras clave: redes globales de producción, aprendizaje tecnológico, empresarios locales, industria automotriz.

\begin{abstract}
Within the context of the severe crisis faced by the U.S. based automotive firms, one of their strategies has been the relocation of the assembly plants in Mexico. This article analyzes the case of the automotive cluster led by Ford in Hermosillo, in the Mexican state of Sonora, showing that this type of clusters led by transnational corporations create technological spillovers and stimulate the accumulation of local capabilities. Particular attention is given to the process of creation of local small and medium enterprises linked to global production networks.

Keywords: global production networks, technological learning, local entrepreneurship, automotive industry.
\end{abstract}

* Este artículo tiene su origen en el proyecto "Estudio sobre el impacto de la ampliación de la Ford Motor Co. en Hermosillo, Sonora", realizado por la Fundación México-Estados Unidos para la Ciencia y El Colegio de Sonora por encargo de la Secretaría de Economía y bajo la coordinación de Óscar F. Contreras. Los autores agradecen la colaboración de Luis Munguía y Paola Millán, así como de todos los integrantes del Seminario Permanente sobre Aprendizaje Tecnológico, Instituciones y Desarrollo Local (Colson-Unison-ITH).

** Investigadores del Centro de Estudios de América del Norte de El Colegio de Sonora. 


\section{Introducción}

En el marco de las nuevas estrategias de la industria automotriz global, México ha asumido un papel cada vez más relevante en la manufactura de automóviles para la región de América del Norte; en particular, el país se ha convertido en una plataforma de producción disputada por las firmas estadounidenses y asiáticas, en su encarnizada competencia por la supremacía en el mercado de América del Norte.

La ampliación de la planta Ford de Hermosillo (2004) y el lanzamiento de la Plataforma CD3 (2005) son parte de la estrategia de Ford para el mercado de autos subcompactos en América del Norte. Se trata de una pieza clave de su estrategia para enfrentar la intensa competencia de las firmas asiáticas, muy particularmente de modelos como el Honda Accord y el Toyota Camry. La estrategia contempla a la vez reducir costos, elevar la calidad de sus productos y recuperar participación en el difícil mercado de América del Norte.

Desde el punto de vista local, el lanzamiento de los nuevos modelos basados en la Plataforma CD3 en Hermosillo (entre ellos el Ford Fusion) representó una enorme derrama económica, ya que la ampliación de la planta armadora y la construcción de un nuevo parque de proveedores requirieron de una inversión superior a los 1,700 millones de dólares. Pero además reactivó una vieja discusión, que involucra tanto a los círculos políticos y empresariales como a los académicos, en torno a las transnacionales y sus operaciones en México: ¿se benefician las regiones receptoras con las inversiones de las transnacionales? ¿Pueden las empresas locales participar en sus cadenas de proveedores? ¿Por qué es tan pobre la incorporación de insumos y componentes locales en las manufacturas de exportación?

En opinión de numerosos actores regionales, las nuevas inversiones de Ford y sus grandes proveedores transnacionales amplían a una escala sin precedentes las oportunidades de negocios, además de que la región parece estar ahora en condiciones de aprovechar la experiencia acumulada durante casi 20 años de familiaridad con la industria automotriz. En este trabajo abordamos uno de los aspectos involucrados en este debate, el que atañe a la participación de los empresarios regionales (entendidos aquí como originarios o residen- 
tes en el estado de Sonora) en las nuevas oportunidades de negocios abiertas por la expansión de la industria automotriz.

\section{Empresas transnacionales, proveedores locales}

La incorporación de las pequeñas y medianas empresas locales en las redes de proveedores de las empresas globales es un tema de gran relevancia en la actualidad, en la medida en que las grandes firmas transnacionales ocupan un lugar cada vez más dominante y los países en desarrollo enfrentan crecientes dificultades para insertarse exitosamente en la economía global. Para México se trata de un tema clave, pues durante los últimos 25 años el país ha carecido de una política industrial que comande la transición hacia un modelo industrial avanzado, capaz de estimular los segmentos de alto valor agregado. Diversos estudios han mostrado que en la medida en que se establecen en el país empresas transnacionales con procesos de manufactura avanzada se transfieren más funciones productivas y gerenciales a las plantas locales, pero hasta ahora ha sido un proceso difuso que no ha generado una base local de proveedores especializados en productos de alto valor agregado o intensivos en conocimiento (Lara, 1998; Carrillo y Hualde, 1998; Contreras, 2000; Dutrénit et al., 2006).

Los estudios sobre la derrama tecnológica de las empresas transnacionales (ETN) y su impacto en la acumulación de capacidades locales se han desarrollado notablemente desde finales de los años ochenta en México (Juárez, Lara y Bueno, 2005; Villavicencio, 2006; Solleiro, 2006). Los estudios recientes discurren en tres vertientes relativamente complementarias: el enfoque del aprendizaje tecnológico (Dutrénit et al., 2006; Lara, 2007; Carrillo, 2004); el enfoque de las redes de conocimiento, basado en el concepto de sistema de innovación (Casas y Luna, 2001); y el enfoque del entorno institucional (Villavicencio, 2006; Casalet, 2004).

En su estudio sobre las maquiladoras electrónicas de Ciudad Juárez, Dutrénit y sus coautores encontraron que la acumulación local de capacidades tecnológicas no es necesariamente un resultado deliberado de estrategias corporativas para construir capacidades tecnológicas descentralizadas en una lógica vertical, sino que intervienen factores como 
[...] el esfuerzo de los gerentes de las plantas locales para atraer nuevos productos, negocios o actividades técnicas, y [...] las diferencias en el poder de negociación de esos gerentes locales. [Estos] factores generan presiones sobre el corporativo para reconocer las capacidades tecnológicas acumuladas localmente y permitirles desarrollar actividades técnicas de mayor innovatividad (Dutrénit et al., 2006:183).

Por su parte, Casas y Luna identifican en diversas regiones del país una creciente transferencia de flujos de conocimiento entre distintos actores e instituciones, que dan lugar a la construcción de redes de conocimiento y espacios regionales de conocimiento. "La formación de estos espacios está conduciendo a la recombinación de conocimientos entre las universidades y los centros públicos de investigación y a través de sus relaciones con las empresas, lo que favorece la creación de masas críticas de investigadores en campos de desarrollo tecnológico especializado" (Casas y Luna, 2001:15).

La tercera vertiente ha mostrado que, además del aprendizaje tecnológico, la trayectoria industrial de algunas regiones ha favorecido la acumulación de capacidades institucionales orientadas a la promoción del desarrollo industrial. A lo largo de la última década surgieron diversas instituciones locales, que algunos autores denominan "instituciones puente"; éstas son "organizaciones que actúan como enlace y apoyo de la producción. La función que desempeñan se vincula con la generación de un entorno de confianza y certidumbre al armar redes de colaboración entre diferentes actores facilitando la interacción y el aprendizaje" (Casalet, 2004:60). En un estudio sobre dos localidades del norte de México se concluye que estas instituciones son producto de iniciativas de los propios actores locales a partir de un real interés en buscar nuevas oportunidades y soluciones para sus regiones, y que han adquirido un papel protagónico en sus respectivos entornos a través de servicios de información, capacitación, financiamiento, organización de eventos y coordinación de actividades, entre otros (Moloman, 2006).

Los estudios mencionados aportan valiosos elementos para analizar el proceso de acumulación de capacidades a través de la interacción 
de los agentes e instituciones locales con las empresas transnacionales. Sin embargo, en esos trabajos el tema de la creación de empresas locales vinculadas a las transnacionales permanece como un aspecto marginal.

Un enfoque que puede enriquecer la comprensión de este fenómeno es el de las Redes Globales de Producción (RGP). Esta perspectiva se interesa por la lógica de la organización productiva en el plano global, enfatizando las interacciones y la articulación entre las empresas líderes globales y las empresas locales a lo largo de las cadenas productivas. Sin perder de vista las relaciones de poder que subordinan a los agentes locales, una de las virtudes de este enfoque es que a la vez permite evaluar las capacidades de los actores locales para influir en la configuración de las redes (Ernst, 2000; Gereffi, 1999; Schmitz, 2004). Si bien las redes son jerárquicas y generalmente están comandadas por las transnacionales, las empresas líderes deben proveerse de capacidades especializadas fuera de la firma como una vía para enfrentar las intensas presiones competitivas del mercado global; tales capacidades pueden abarcar desde una simple subcontratación de la fase de ensamble hasta sofisticados procesos de diseño (Ernst y Kim, 2002). Así, las empresas transnacionales necesitan transferir capacidades técnicas y gerenciales a sus filiales y proveedores locales para que éstos sean capaces de cumplir con sus exigentes estándares de calidad y a la vez disminuir los costos de producción. Una vez que han logrado elevar sus capacidades, los nuevos estándares alcanzados se convierten en un incentivo para delegar conocimientos y procesos más sofisticados en los proveedores locales.

Desde el punto de vista de las empresas locales, hay tres aspectos que definen la racionalidad fundamental de las redes globales de producción: en primer lugar, permiten a las empresas líderes mantener su competitividad global, asegurando su acceso a proveedores especializados en regiones de bajos costos que además son capaces de ofrecer respuestas rápidas y flexibles a sus requerimientos de insumos, servicios y fuerza de trabajo.

En segundo lugar, las redes actúan como medios de transferencia de conocimiento desde las empresas líderes hacia los proveedores locales. Esto es necesario para mejorar las habilidades técnicas y admi- 
nistrativas de los proveedores, de modo que puedan alcanzar las especificaciones técnicas de las líderes. Una vez que un proveedor logra mejorar satisfactoriamente sus capacidades, se crea un incentivo para que las líderes transfieran conocimientos más sofisticados, incluyendo la ingeniería de productos y procesos y eventualmente incluso actividades de diseño.

Por último, los proveedores locales sólo pueden absorber efectivamente el conocimiento diseminado por las líderes en la medida en que han desarrollado sus propias capacidades. La efectividad y la velocidad de la conversión del conocimiento dependerá no tanto de la cantidad y calidad del conocimiento trasferido por las líderes sino de la capacidad de absorción de las proveedoras locales. La dotación inicial de conocimiento local determina el nivel de sofisticación del conocimiento transferido, mientras que la intensidad del esfuerzo acelera la velocidad de los procesos de conversión (Ernst y Kim, 2002).

El enfoque de las RGP ofrece así un marco analítico apropiado para captar la lógica y analizar los mecanismos de aprendizaje tecnológico y la acumulación de capacidades que permiten a las empresas locales participar en las redes de abastecimiento de las transnacionales. No se trata solamente de un problema de voluntad de los empresarios locales o de las restricciones a la entrada impuestas por las transnacionales, sino de una compleja articulación entre las estrategias globales de las empresas líderes y los procesos de creación de capacidades locales.

\section{Los empresarios regionales y la globalización}

Sonora es uno de los estados fronterizos del norte de México que, en virtud de su condición geográfica, ha mantenido una estrecha vinculación con la economía de los Estados Unidos. Con una fuerte tradición agrícola y agroindustrial, en el último tramo del siglo XX el estado experimentó un proceso de industrialización impulsado por la inversión extranjera y basado principalmente en el establecimiento de plantas maquiladoras (Ramírez, 1988; Vázquez, 1991; Wong, 1996). Las nuevas actividades industriales configuraron un nuevo mapa regional, al pasar de una estructura centrada en el sector primario y con 
una importante participación de capitales locales, hacia una economía engarzada a la lógica de producción global presidida por un sector industrial con muy escasos vínculos regionales y con asiento principal en las zonas fronterizas y en la capital del estado (Contreras y Rodríguez, 2003).

El cambio estructural representado por el tránsito de la economía primaria a la industrial implicó que los agentes locales fueran desplazados del papel estratégico que antaño tuvieron en la conducción de la economía regional. La nueva industrialización recibió su impulso del exterior y en virtud de ello la región asumió un nuevo papel como eslabón manufacturero en cadenas globales de producción comandadas por las transnacionales, principalmente de las ramas electrónica y automotriz.

Los efectos de esta reestructuración de la economía regional implicaron la ruptura de un tejido social y económico tradicional, tal como lo admiten casi todos los analistas del periodo. Sin embargo, la interpretación del sentido de tales impactos acepta diferentes enfoques. Por una parte desplazó a los empresarios locales del protagonismo económico que tuvieron durante décadas, confinándolos a un papel de espectadores de la nueva estrategia de desarrollo (Wong, 1993; Vázquez y García, 1992). Pero también tuvo otro tipo de impactos como la ampliación del mercado de trabajo para un segmento de trabajadores y empleados altamente calificados (Contreras y Rodríguez, 2000), y la apertura de nuevos campos de acción para negocios locales, como lo ejemplifica el caso de las empresas vinculadas a las nuevas tecnologías de la información (Contreras, Félix y Ponce, 2001).

Al momento de entrar en vigor el Tratado de Libre Comercio de América del Norte (TLCAN) en 1994, la transición hacia la economía industrial basada en manufacturas de exportación estaba prácticamente concluida, y eran este tipo de actividades ligadas al capital transnacional las que definían los principales indicadores de desempeño económico regional. Ya para el final de la década las actividades más altamente exportadoras eran las manufacturas modernas; las maquiladoras y la industria automotriz representaban más del 75\% del valor de las exportaciones totales, si bien algunos segmentos primarios recobraron presencia como altamente exportadores. 
La entrada en vigor del TLCAN desencadenó una reactivación general de la economía regional: se incrementó de manera drástica la inversión extranjera, las exportaciones, el saldo positivo en la balanza comercial y se generaron más empleos que en el periodo anterior al Tratado; al mismo tiempo, sin embargo, el TLCAN reforzó decisivamente las tendencias a la polarización de la economía regional y a la integración del espacio económico sonorense como una plataforma manufacturera de la economía estadounidense.

En el nuevo modelo la región asumió un moderno papel como eslabón manufacturero en cadenas globales de producción comandadas principalmente por las ramas electrónica y automotriz. Esta reestructuración supuso la ruptura del tejido social y económico tradicional, dando paso a un modelo económico polarizado en el que la mayoría de las empresas regionales, severamente golpeadas por la apertura comercial, quedaron marginadas del ciclo de crecimiento favorecido por el TLCAN. Pero a la vez este proceso amplió el mercado de trabajo para un segmento de trabajadores y empleados altamente calificados y abrió nuevos campos de acción para la nueva generación de empresarios y de negocios locales. De esta manera las transnacionales y sus socios locales, así como un puñado de empresas regionales que se ajustaron a las nuevas condiciones, se convirtieron en los actores protagónicos de este nuevo episodio de la historia económica regional.

\section{Los empresarios sonorenses}

Los estudios disponibles acerca del desarrollo y desempeño de los grupos empresariales sonorenses coinciden en señalar que en su surgimiento y consolidación fueron determinantes los vínculos cultivados con el poder político (Vázquez y Hernández, 2001).

En la consolidación de la clase empresarial regional después de la Revolución mexicana un factor clave fue la estrecha relación entre los empresarios y los líderes de la fracción triunfadora, el llamado "Grupo Sonora". Ese grupo, encabezado por Álvaro Obregón, Plutarco Elías Calles, Adolfo de la Huerta y Abelardo L. Rodríguez, tuvo un papel protagónico en la política nacional por espacio de 20 años, lapso en el 
que ejercieron su influencia para desarrollar la agricultura comercial en el sur del estado de Sonora. Para ello se canalizaron cuantiosos recursos fiscales en infraestructura, obras hidráulicas, caminos vecinales, y apertura de nuevas tierras a la explotación.

Sonora se transformó en una moderna región agrícola (en su momento llegó a ser llamada "el granero de México") en buena medida al amparo de la inversión pública y bajo la protección directa del grupo gobernante. Éste es un elemento medular en el comportamiento posterior del empresariado regional, que al establecerse en actividades de alta rentabilidad y con fuerte apoyo de la inversión pública desarrolló poca proclividad a la inversión de riesgo y encontró escasos incentivos para incursionar en actividades manufactureras.

Gracias a esas inversiones, en poco tiempo la economía sonorense transitó del modelo minero-pecuario predominante al inicio del siglo XX, hacia un modelo de desarrollo dominado por una floreciente producción agrícola moderna. Esta transformación implicó la redefinición de prácticamente todas las actividades económicas para insertarse en el funcionamiento del nuevo patrón de acumulación.

En la actualidad la estructura productiva de Sonora ha dejado de descansar en la actividad agropecuaria, y las relaciones entre los empresarios y la clase política son más difusas. Desde los años setenta del siglo pasado (con el establecimiento de las primeras maquiladoras), pero sobre todo a partir de la segunda mitad de los ochenta (con el establecimiento de la planta de estampado y ensamble de Ford en Hermosillo), la industria manufacturera desplazó al sector primario como la actividad económica predominante y se convirtió en el motor del dinamismo económico de Sonora. Se trata de un sector dominado por empresas extranjeras ligadas a redes de producción global, tales como las maquiladoras electrónicas, de autopartes y, más recientemente, de la industria aeroespacial, así como la propia planta Ford y sus empresas proveedoras. Este tipo de inversiones exige largos periodos de maduración y requiere de capacidades tecnológicas y empresariales de nivel global.

En estas condiciones no es extraño que los legendarios agrotitanes de la región hayan sido desplazados por empresarios foráneos, extranjeros o nacionales, mismos que asumieron el timón de la indus- 
trialización regional. Los empresarios locales no estaban preparados para desempeñarse con éxito en el ambiente altamente competitivo y tecnológicamente sofisticado de la manufactura moderna. Empresarios de larga tradición como los Valenzuela, Bórquez, Gutiérrez, Salido, Hoeffer, Camou, Mazón, Gaxiola, Elías, entre otros, que dominaron el mundo empresarial de la región desde los años treinta hasta los ochenta del siglo XX, ahora se dedican a actividades complementarias o secundarias a los nuevos ejes dinamizadores de la economía regional, como por ejemplo la construcción de naves industriales, la venta de terrenos o los servicios tipo shelter para las maquiladoras.

Por otro lado, la economía sonorense se ha diversificado. A la expansión de la industria manufacturera se une un crecimiento inusitado del comercio con el arribo de cadenas de autoservicio nacionales y extranjeras, mismas que han desplazado a las firmas nativas (Bocanegra, 2005). De igual forma la agricultura tradicional (centrada en cultivos básicos) pierde relevancia en favor de cultivos de alto valor agregado (como frutales y hortalizas) destinados primordialmente al mercado de los Estados Unidos (Valenzuela y Bracamonte, 2005). La minería en los últimos diez años ha confirmado su naturaleza cíclica: combina años de crecimiento acelerado, con periodos prolongados de desaceleración que conducen a la devastación de pueblos enteros. La pesca, y en especial la acuacultura, se están expandiendo notablemente (Méndez y Bracamonte, 2005).

En este contexto, hacia finales del 2003 el anuncio de la ampliación de la planta Ford de Hermosillo y el establecimiento de una veintena de grandes proveedores transnacionales de autopartes alimentó grandes expectativas. Tanto en los círculos empresariales como en los medios gubernamentales se generó un clima de optimismo bajo el supuesto de que el nuevo conglomerado automotriz daría un renovado impulso a la economía regional e iniciaría una nueva fase de desarrollo manufacturero que permitiría al empresariado local involucrarse en la proveeduría del cluster automotriz. ${ }^{1}$

${ }^{1}$ Como ejemplo de ese clima se puede mencionar que durante un año, entre 2004 y 2005, el diario El Imparcial (el de mayor circulación en el estado) dedicó un suplemento especial de una plana semanal a promover las oportunidades de negocios relacionadas con la ampliación de la planta Ford. 
El examen de la trayectoria de los grupos locales muestra, sin embargo, que la posibilidad de desarrollar una red local de proveedores de la industria automotriz pareciera no estar al alcance de estos empresarios, debido a que durante las dos décadas previas (que comprenden el periodo de mayor expansión de las maquiladoras y el establecimiento de la planta Ford en Hermosillo) los empresarios regionales no desarrollaron capacidades para emprender proyectos de base tecnológica ni para cumplir con los estándares de calidad exigidos por las transnacionales.

Una aproximación a esa problemática se ilustra con algunos datos de las más grandes empresas registradas en Sonora. Entre las decenas de grandes corporativos con actividades en el estado, sólo unos cuantos son propiedad de empresarios sonorenses y de ellos ninguno tiene vínculos directos con la industria automotriz o con procesos intensivos en conocimiento (cuadro 1).

En el cuadro 1 se puede apreciar que una parte importante del empresariado regional sigue operando en negocios ligados a las actividades primarias. ${ }^{2}$ Prácticamente todos los empresarios incluidos en el cuadro iniciaron en la agricultura, y el que ahora lo hagan en la industria alimentaria refleja una trayectoria natural de escalamiento en la propia cadena productiva. Las firmas incluidas son las empresas de capital sonorense de mayor tamaño establecidas en la entidad; pese a ello no necesariamente se trata de las firmas más emprendedoras en cuanto a su disposición para incursionar en actividades manufactureras o de servicios tecnológicos que les permitiesen participar en los nichos de alto valor agregado abiertos por la expansión de la industria automotriz.

\section{Una tipología de los grupos empresariales}

En el marco de las transformaciones experimentadas por la economía sonorense a lo largo del siglo XX, una primera clasificación permite

\footnotetext{
${ }^{2}$ Varios grupos que en el propio medio empresarial son reconocidos por su importancia no aparecen en el listado, probablemente debido a que sus capitales están diseminados en numerosas empresas de tamaño mediano. Es el caso de la
} 


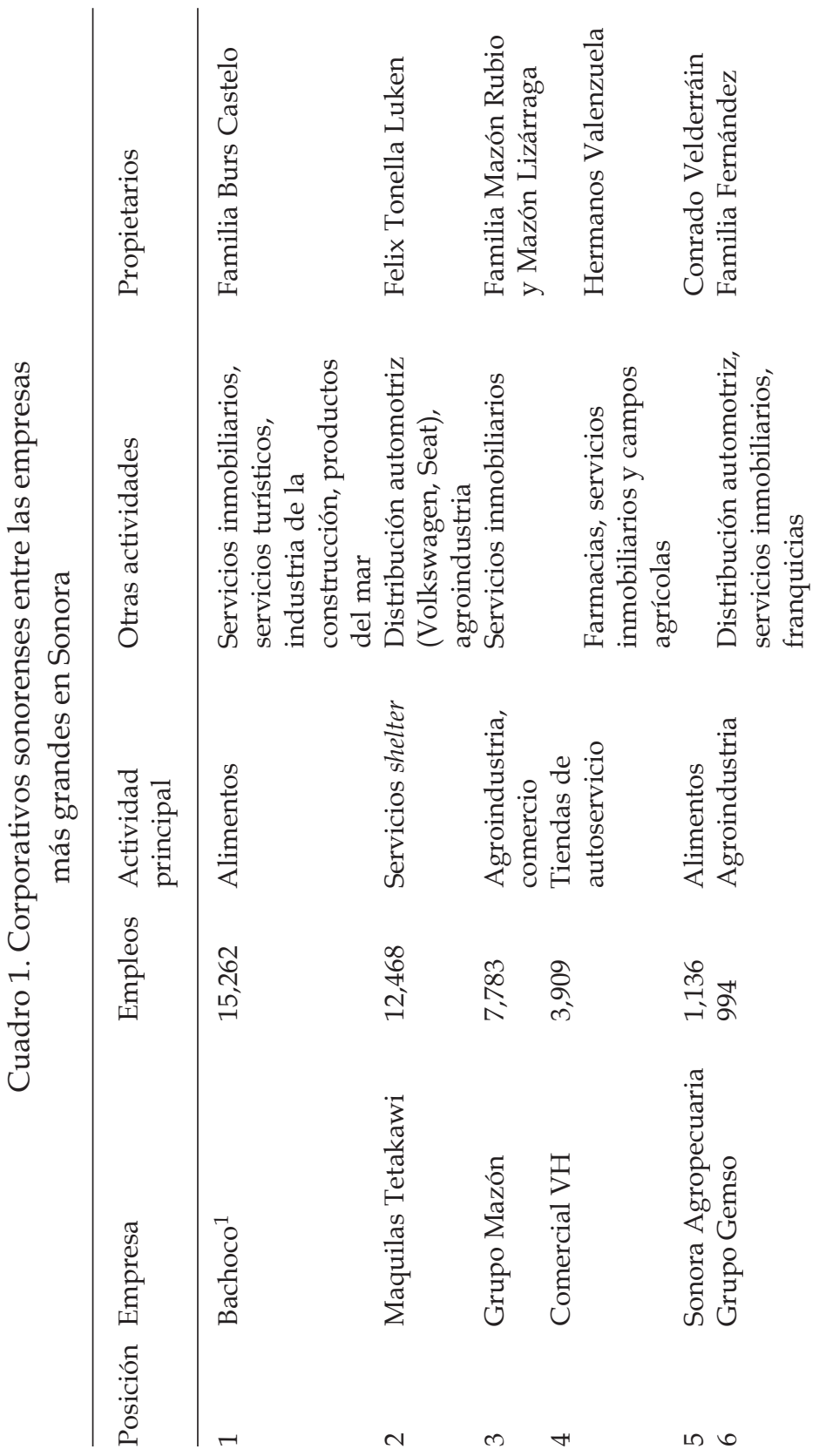




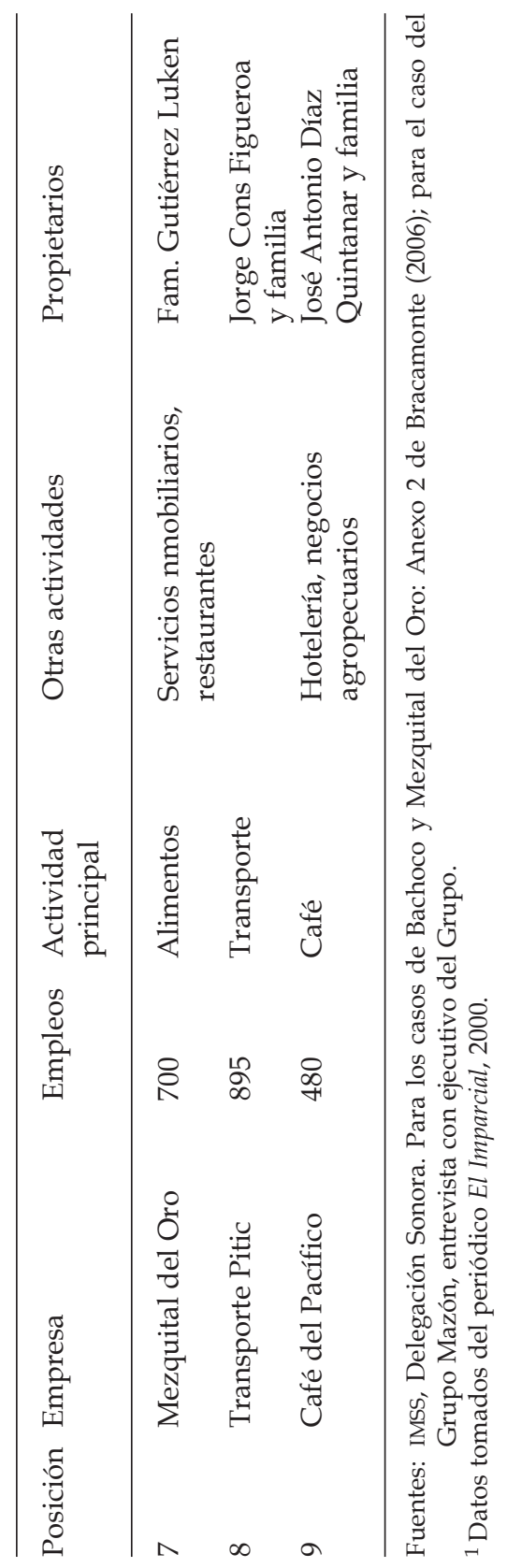


identificar al menos cuatro grupos empresariales en el estado. ${ }^{3}$ En primer lugar están los agricultores tradicionales de los valles que no pudieron enfrentar las nuevas reglas de la apertura comercial en virtud de que no diversificaron sus actividades. Un segundo grupo está conformado por los empresarios agrícolas que se diversificaron hacia el comercio, la banca y la industria pero que vieron mermada su competitividad una vez puesta en marcha la apertura comercial. En este caso algunos ganaron pero la mayoría vio disminuir su influencia y poder económico.

En la tercera categoría se ubican los empresarios modernos, un grupo formado por hijos de los antiguos empresarios tradicionales. Se trata de una generación dedicada a negocios vinculados con la urbanización e industrialización (principalmente en empresas constructoras que edifican naves e incluso parques industriales para ponerlos a la disposición de inversionistas manufactureros foráneos).

Hay un cuarto segmento de empresarios de origen diverso cuyo rasgo principal es ser "facilitadores de la integración". Estos empresarios aprovecharon la modernización y reestructuración productiva para incursionar en actividades directamente ligadas a la expansión industrial, tales como el transporte de carga, los negocios tipo shelter e incluso las manufacturas; son exportadores que supieron usar las ventajas de localización de un estado cuyo crecimiento económico en las últimas décadas ha dependido principalmente de su vinculación con la economía de Estados Unidos.

El cuadro 2 incluye a algunas de las empresas más características de cada una de estas categorías. ${ }^{4}$

familia Fernández Aguilar, cuyos negocios abarcan actividades agroindustriales, servicios financieros e inmobiliarios, y que por cierto es uno de los casos excepcionales de empresarios regionales cuyas actividades originales se han diversificado hasta abarcar la manufactura de autopartes.

${ }^{3}$ Esta clasificación se basa en el trabajo de Vázquez y Hernández (2001); la que aquí proponemos es una versión modificada de la que elaboraron estos autores.

${ }^{4}$ Adicionalmente estarían las firmas externas, nacionales o internacionales, que son las que desde el inicio del proceso de industrialización se apoderaron y controlaron los segmentos más dinámicos de la economía regional, sobre todo en la manufactura, el comercio a gran escala y en menor medida en la minería 
Cuadro 2. Clasificación de los grupos empresariales sonorenses

\begin{tabular}{lll}
\hline Categoría & Principales actividades & Empresarios \\
\hline $\begin{array}{l}\text { Empresarios agrícolas } \\
\text { tradicionales }\end{array}$ & Ganadería, alimentos & $\begin{array}{l}\text { Marco Antonio Molina } \\
\text { Conrado Velderrain }\end{array}$ \\
$\begin{array}{l}\text { Empresarios agrícolas } \\
\text { diversificados }\end{array}$ & $\begin{array}{l}\text { Agroindustria, servicios } \\
\text { inmobiliarios, comercio }\end{array}$ & $\begin{array}{l}\text { Roberto Mazón Rubio } \\
\text { Javier Gutiérrez Luken }\end{array}$ \\
Empresarios modernos & $\begin{array}{l}\text { Agroindustria, servicios } \\
\text { financieros, alimentos, } \\
\text { electrónica }\end{array}$ & $\begin{array}{l}\text { Benjamín Aguilar } \\
\text { Faustino Fernández Aguilar }\end{array}$ \\
$\begin{array}{l}\text { Facilitadores de la } \\
\text { integración }\end{array}$ & $\begin{array}{l}\text { Servicios shelter, } \\
\text { transporte de carga }\end{array}$ & $\begin{array}{l}\text { Félix Tonella Luken } \\
\text { Jorge Cons Figueroa }\end{array}$ \\
\hline
\end{tabular}

Fuente: Adaptado con base en Vázquez y Hernández (2001).

Para este trabajo se entrevistaron siete empresarios que cubren las cuatro categorías de la clasificación contenida en el cuadro: empresarios agrícolas tradicionales (Marco Antonio Molina); empresarios agrícolas diversificados (Manuel Pedroza); facilitadores de la integración (Jorge Cons y Félix Tonella) y empresarios modernos (Faustino Fernández, José Antonio Díaz Quintanar y Benjamín Aguilar). ${ }^{5}$

La selección se realizó tomando como base la clasificación de Vázquez y tomando como criterio principal el tamaño del grupo empresarial al que encabezan; así, seis de los empresarios seleccionados figuran como propietarios de otras tantas firmas ubicadas entre las más grandes del estado: Transportes Pitic (Jorge Cons), Maquilas Tetakawi (Félix Tonella), Café del Pacífico (José A. Díaz Quintanar), Grupo Mazón (Familia Mazón Rubio y Mazón Lizárraga), Sonora Agropecuaria (Conrado Velderráin) y Mezoro (Familia Gutiérrez Luken).

Las familias Mazón y Gutiérrez Luken cumplen a su vez con una característica subrayada por Vázquez (2001): son empresarios en plena actividad y herederos de los agrotitanes de la post-revolución. Ade-

\footnotetext{
${ }^{5}$ En los casos de los empresarios agrícolas diversificados no fue posible entrevistar a Gutiérrez Luken ni a Roberto Mazón. En cambio se entrevistó a un alto ejecutivo integrante del Consejo Administrativo.
} 
más se incluye a un grupo que sin encabezar grandes corporativos son considerados en la región como "empresarios regionales exitosos". Se incluyeron en esta categoría a Faustino Fernández, un activo promotor de la cultura productiva moderna, además de ser él mismo un próspero empresario; Marco Antonio Molina, uno de los agricultores regionales más exitosos que además ha ejercido diversos cargos como dirigente empresarial; ${ }^{6}$ y Benjamín Aguilar, copropietario de Lanix, empresa ensambladora de computadoras personales y portátiles, un caso excepcional de una empresa de base tecnológica que se ha mantenido y expandido en un mercado sumamente competido.

Con base en el análisis de contenido de las entrevistas, se elaboró una nueva tipología de los grupos empresariales de la región, tomando como eje analítico la participación de los empresarios en actividades industriales (cuadro 3):

Empresarios primarios. Se trata de un grupo dedicado desde su origen a actividades agropecuarias, que no ha expandido sus actividades a la industria. Exhiben una relativa diversificación pero en un ámbito limitado a los sectores primarios de la economía y en menor escala en los servicios. De cualquier forma, son empresarios cuya explotación agrícola se realiza empleando tecnologías modernas. Los procesos que desarrollan, los montos de inversión exigidos, así como los riesgos en que incurren, les hacen estar familiarizados con los mercados modernos y con las nuevas tecnologías, aunque sus capacidades empresariales y tecnológicas se ubican en segmentos muy lejanos de las manufacturas globales.

Empresarios agroindustriales. Los negocios de este tipo de empresarios se concentran en el procesamiento y la transformación de commodities, pero también la agroindustria es la actividad seminal de la trayectoria empresarial del grupo. Las generaciones herederas se involucraron posteriormente en el sector agropecuario (ganadería y agricultura) y en los servicios. Las empresas lograron desarrollar capacidades tecnológicas avanzadas, vinculadas

${ }^{6}$ Actualmente es el presidente de la Fundación Produce. Antes era el líder de los agricultores de la costa de Hermosillo. 


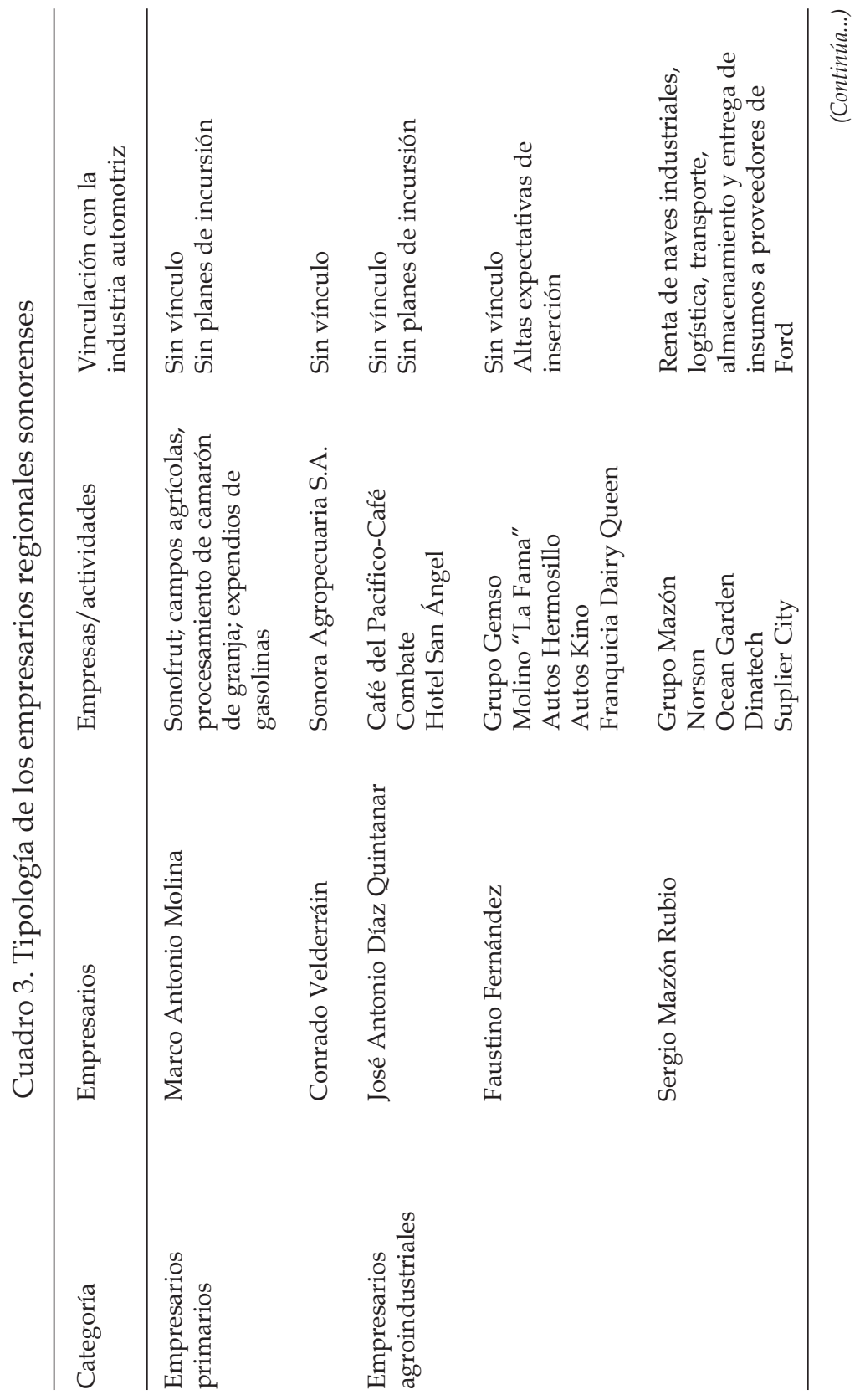




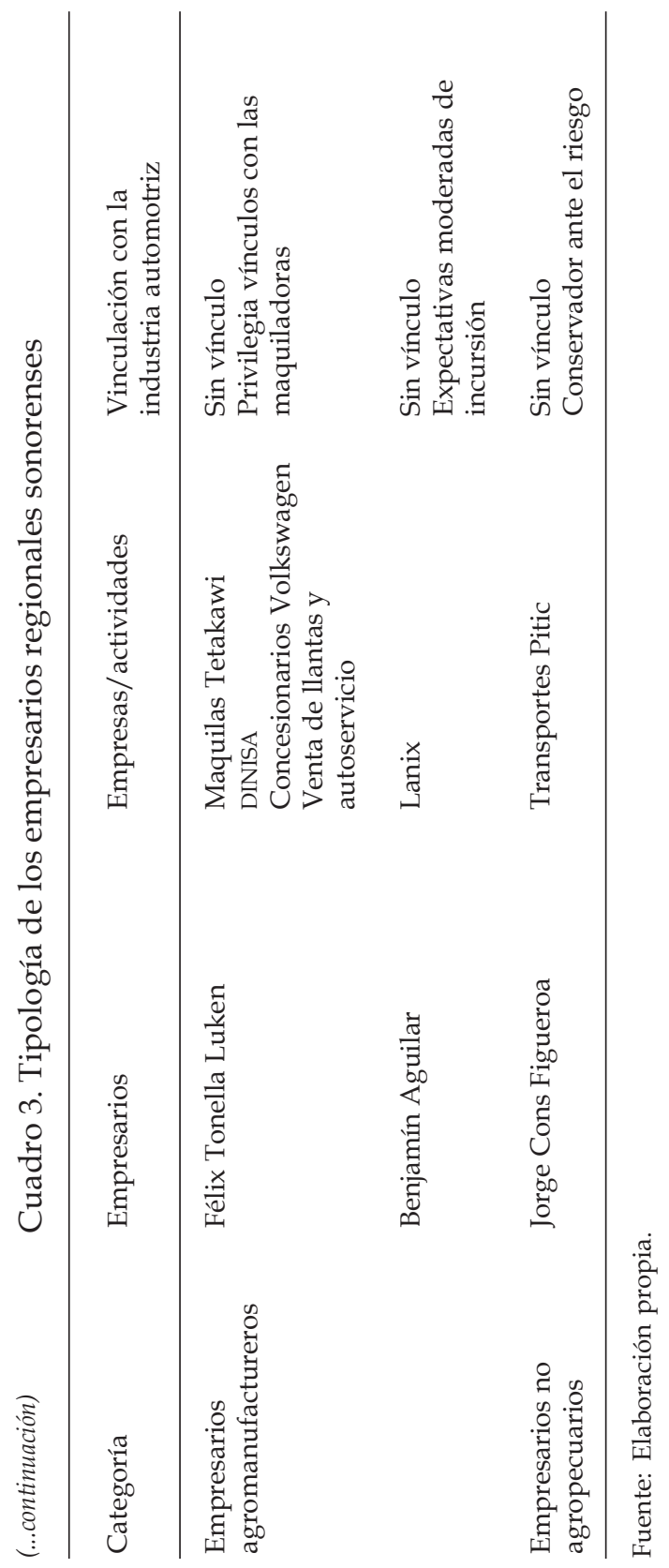


a las actividades agroindustriales, así como una creciente familiaridad con los mercados internacionales.

Empresarios agromanufactureros. La trayectoria empresarial de este grupo inicia en las actividades primarias, que aún mantienen en alguna medida, pero sus actividades predominantes se relacionan en la actualidad con la industria manufacturera, sobre todo porque se trata de un grupo de empresarios que en el redespliegue industrial de los años setenta y ochenta -expansión de la industria maquiladora, crecimiento de actividades periféricas a la industria automotriz y emergencia de nuevas actividades ligadas a la economía de la información- aprovecharon distintos nichos de oportunidades en el sector manufacturero, convirtiéndolo en la columna vertebral de sus negocios. Se trata de empresas que no están ligadas a la industria automotriz pero que han desarrollado líneas de producción complejas.

Empresarios no agropecuarios. Se incluye en esta categoría a aquellos empresarios cuyo origen no está asociado a las actividades agrícolas sino a negocios urbanos y directamente ligados a la expansión de las actividades industriales durante las últimas dos décadas; se trata de empresas que utilizan tecnologías y prácticas organizacionales modernas y que han logrado incursionar en los mercados internacionales, si bien sus propias actividades no son industriales sino vinculadas a servicios de logística, transporte y administración.

A continuación se presentan siete breves perfiles de los empresarios entrevistados, incluyendo comentarios sobre sus percepciones y expectativas en torno al desarrollo de un sector de alta tecnología vinculado a la expansión de la industria automotriz en la región.

\section{Empresarios primarios}

\section{MARCO ANTONIO MOLINA}

Inició su trayectoria empresarial alrededor de 1966, incursionando en actividades comerciales, en especial ligadas con la venta de maquina- 
ria, equipo agrícola y ferretería. Sus negocios llegaron a abarcar diversas regiones a lo largo del estado de Sonora, e incluso estableció sucursales en estados vecinos.

Para 1980, debido a los cambios en materia de política agrícola que significaron una notable reducción de los apoyos gubernamentales al campo, muchos productores agrícolas dejaron la actividad. Esta baja en el dinamismo abrió un nicho de oportunidad y fue entonces cuando este empresario decidió invertir precisamente en esta actividad para diversificar sus cultivos, incursionando en productos de exportación, principalmente uva y hortalizas.

Debido a la crisis de 1994 y a problemas de pasivos, se vio obligado a hacer algunos reajustes en sus negocios, dando un giro hacia la actividad inmobiliaria (compra-venta de terrenos), sin dejar de lado la actividad agrícola. Recientemente ha incursionado en otras actividades relacionadas con la agricultura (mediante la empresa Sonofrut), específicamente en servicios de empaquetado, refrigeración y almacenaje de frutas y verduras. También participa en el sector acuícola en actividades de empaquetado y congelado (maquetas para Ocean Garden).

En su visión de negocios, el sector agropecuario debe diversificarse y escalar en la cadena de valor. Esto es, se deben aprovechar las oportunidades de producción primaria con que cuenta el estado y ligarlas a los segmentos industriales y de servicios relacionados al sector agropecuario. De la actividad agropecuaria surgirán nuevas áreas de oportunidad como el empaque, los servicios de logística y la comercialización, entre otras. Una de las actividades que tendrá una participación creciente es la camaronicultura, segmento que hace tiempo era considerado como una actividad sin futuro y que en los últimos años ha mostrado ser muy rentable.

En relación con la industria automotriz, su perspectiva es que a pesar de la importancia que a lo largo de los últimos años ha tenido para el estado la presencia de la planta Ford en Hermosillo, así como de la relevancia de su reciente ampliación, para las empresas regionales resulta prácticamente imposible tener participación directa en esta industria, ya que estas grandes empresas vienen con la proveeduría muy establecida, lo cual constituye una gran barrera para los empresarios locales. 
Sin embargo, aun considerando lo cerrado de estas redes, existe una serie de actividades periféricas que pueden ser desarrolladas por la iniciativa local, tales como la logística, construcción de infraestructura, tareas de mantenimiento y servicios. La mira puede ser hacia los proveedores de Ford: aprovechar el conocimiento local y la infraestructura y conocimiento del empresariado local. Existe además un proceso de tipo generacional, de manera que la inserción del capital sonorense en las empresas de base tecnológica es una posibilidad a futuro y a cargo de las nuevas generaciones, que puedan aprovechar las oportunidades de negocio que se abrirán para el estado con este tipo de industrias.

\section{Empresarios agroindustriales}

\section{José Antonio díaz Quintanar (CAFé del PaCifico-Café COMBATE)}

La empresa tiene su origen en Chihuahua como parte del proceso de expansión de "Tostadores...", si bien en la actualidad Café del Pacífico es una empresa completamente independiente. Fue en la década de los cuarenta del siglo XX cuando fundaron la empresa, actualmente administrada por la tercera generación de empresarios familiares. El grupo se ha diversificado a otras actividades como las bienes raíces, la ganadería, el turismo y la acuacultura. Café Combate es la columna vertebral del grupo pues factura la mayor parte de sus ingresos.

En relación con la industria automotriz el empresario tiene una visión escéptica. En particular su grupo no está involucrado en actividades relacionadas con Ford, ni tiene planes para involucrarse en el futuro. En su opinión los inversionistas no se arriesgan en proyectos de inversión de base tecnológica debido a que no tienen la experiencia ni la capacidad para incursionar en ese tipo de actividades.

\section{FAUSTINO FERNÁNDEZ (GRUPO GEMSO)}

El Grupo Gemso se dedica a varias actividades, entre las que destacan la agricultura, las bienes raíces, la distribución de autos y la industria- 
lización de harina de trigo, entre otras. A este grupo pertenecen los hermanos Faustino y José Ramón Fernández, además de Javier Gándara. Se trata de uno de los grupos más afianzados en la comunidad empresarial sonorense; de hecho mantienen una tradición de participación en la vida pública local, a través de asociaciones civiles tales como Impulsa, dedicada a impartir cursos de desarrollo empresarial en todos los niveles del sistema educativo local. Lo mismo hacen Javier Gándara y su esposa Marcela Fernández mediante agrupaciones altruistas y sobre todo a través de la fundación Ganfer.

Faustino Fernández es un empresario atípico. Además de los negocios que encabeza dentro del Grupo Gemso, por iniciativa propia desarrolla actividades vinculadas a la industria con una empresa de reconstrucción de partes automotrices cuyas operaciones se extienden por varios estados.

Fernández es uno de los más entusiastas promotores de la modernización empresarial y tiene altas expectativas de lograr insertarse en la cadena productiva de Ford, con base en el análisis de las oportunidades de inversión que se desprenden de la expansión de sus operaciones en Hermosillo. Tiene la convicción de que los empresarios sonorenses están capacitados para aprovechar este tipo de oportunidades, convicción basada en su propia experiencia y en el hecho de que un buen número de empresarios jóvenes desconocidos están promoviendo interesantes iniciativas manufactureras mismas que reúnen la calidad y cantidad necesaria para ser competitivos. Considera que si a estos nuevos empresarios se les diera una oportunidad en la red de suministros de Ford, tendrían éxito.

\section{Manuel Pedroza Andrade (Grupo MaZón)}

El Grupo Mazón representa una de las organizaciones empresariales de mayor arraigo en Sonora. El primero de los negocios de la familia, una tienda de abarrotes de nombre "La Cosalteca", fue creado en Hermosillo hacia 1898 por José T. Mazón Salazar y sus hijos. En 1954 Grupo Mazón, integrado principalmente por la familia Mazón López, expande sus operaciones y abre la primera tienda departamental Mazón, dando inicio al proceso de diversificación de 
sus actividades que en la actualidad incluyen negocios agroindustriales (con empresas como Norson y Ocean Garden), parques industriales, distribución de llantas, servicios automotrices, actividades inmobiliarias y de servicios comerciales, entre otras en las que encuentra una participación accionaría importante. Actualmente el cuerpo directivo está conformado principalmente por la familia Mazón Rubio y Mazón Lizárraga.

Respecto a su inserción en el cluster automotriz, el Grupo Mazón tiene una activa vinculación a través de la empresa Supplier City dedicada a la logística, transporte, almacenamiento y entrega de insumos a proveedores de la planta de ensamble Ford, y Dinatech Sur, empresa que se dedica a la construcción y renta de naves industriales para los proveedores de la planta Ford.

En la actualidad el Grupo Mazón cuenta con más de 7,700 empleos directos en sus diversas actividades y se ha consolidado como una de las empresas sonorenses más duraderas y estables, con una participación cada vez mayor en los mercados internacionales.

\section{Empresarios agromanufactureros}

\section{FÉlix TONELLA (MAQUILAS TETAKAWI)}

El grupo empresarial encabezado por Félix Tonella se constituyó desde su inicio como una empresa de corte familiar donde participan los hermanos, cuñados e hijos de éstos. En la actualidad los negocios son manejados por la tercera generación de descendientes Tonella.

El negocio agrícola lo inició en 1940 el padre de Félix, quien posteriormente se incorpora a los negocios familiares. El buen desempeño de sus negocios en los años setenta posibilitó la incursión en el ámbito inmobiliario (compra-venta de terrenos). Más adelante obtienen la concesión para la distribución de autos de la marca Volkswagen, siendo a la fecha los únicos concesionarios en el estado de Sonora.

En 1988 se estrenan en la actividad manufacturera a través de una empresa tipo shelter que alberga empresas maquiladoras: Maquilas Tetakawi. Actualmente cuentan con tres parques industriales (en Empalme y Guaymas, Sonora y en Saltillo, Coahuila), donde operan 40 
distintas plantas maquiladoras que generan alrededor de 14 mil empleos, de los cuales 12 mil corresponden a Sonora.

La estrategia empresarial de este grupo descansa en tres líneas de acción, mismas que se reflejan en tres diferentes empresas: una ubicada en la ciudad de Tucson, Arizona (matriz), concentra la promoción y contratación del plan shelter; la segunda, llamada DINISA, se encarga de la administración de los parques industriales (naves); y la tercera, Maquilas Tetakawi, realiza la interacción con los clientes.

Entre sus diversificados negocios, el empresario opina que los de mayor potencial a largo plazo son aquellos ligados a la industria maquiladora, pues el estado de Sonora tiene una vocación industrial, aunque no necesariamente en el ámbito de la industria automotriz ya que este tipo de industria requiere de inversiones cuantiosas; además, exige un manejo solvente de la ingeniería, tanto básica como financiera, a lo cual pocos empresarios tienen acceso.

Uno de los efectos positivos de la expansión de la industria automotriz es la adopción de una "cultura del trabajo y del orden". En este sentido, la participación de las empresas locales es en alguna parte de la cadena de suministros y para ello son necesarios análisis y estudios que detecten posibles espacios y nichos de oportunidad.

BENJAMÍN AgUiLAR (LANIX)

Benjamín Aguilar se formó como empresario en una actividad por completo ajena a la industria manufacturera: la porcicultura. En 1987 creó, junto con otro socio que también provenía de una actividad tradicional (la construcción), el grupo empresarial que actualmente fabrica las computadoras Lanix. Dado que el socio tenía previamente negocios en la industria de la construcción, la asociación pronto incluyó también esta actividad.

En 1987 incursionaron en la industria electrónica invirtiendo en la empresa GAMA que en aquel entonces fabricaba computadoras personales. Tres años después, en 1990, se separaron del grupo GAMA para establecer su propia empresa, Lanix. Actualmente Lanix emplea a más de 250 trabajadores en su planta de Hermosillo, ensamblando PC's, portátiles y servidores, con un volumen anual superior a las $60 \mathrm{mil}$ 
unidades, de las cuales sólo el $8 \%$ se vende en el estado. Cuentan con una red de distribución en prácticamente todo el país y en el año 2005 abrieron una planta en Chile con una plantilla de 50 empleados.

El empresario estima que entre sus negocios el que más posibilidades tiene de expandirse es el de la electrónica. Se trata de un mercado donde la competencia es muy severa, pero han acumulado la experiencia suficiente para desempeñarse con éxito. En cuanto a la industria automotriz, su visión es positiva pero estima que se requiere de un esfuerzo empresarial considerable pues este tipo de oportunidades no son fáciles de aprovechar. En particular, la cultura industrial de la región es todavía poco desarrollada, aunque se trata de un aspecto que se puede superar en el corto plazo. Por ejemplo los esquemas de calidad tipo ISO-9000 que le han impreso a las prácticas laborales una cultura de la calidad desconocida hasta hace unos pocos años.

\section{Empresarios no agropecuarios}

\section{Jorge CONS (TRANSPORTES PITIC)}

Jorge Cons es ingeniero mecánico y actualmente director general de Transportes Pitic, una de las principales empresas transportistas en México. Da servicio en todo el territorio nacional, para lo cual cuenta con una flotilla de tractocamiones, remolques y unidades de reparto cercana a las 900 unidades. La facturación es superior a 40 millones de dólares anuales con ritmos de crecimiento superiores al 10\% anual. La nómina es de 895 empleados. Se trata de una empresa familiar que ha podido sortear coyunturas difíciles concentrándose en el servicio al cliente incorporando tecnología en la administración y el control de la flotilla:

[...] hemos procurado siempre tener ventajas competitivas a partir de la tecnología. Así fuimos la primera o la segunda compañía en los noventa en tener una base de datos con todas las operaciones utilizando un sistema que fue desarrollado internamente. Ese sistema se ha perfeccionado; actualmente el cien por ciento de las unidades están equipadas con siste- 
mas de rastreo satelital. Montamos nuestra página Internet para interactuar con los clientes que tienen acceso al estatus de sus embarques directamente por la web. Desde hace más de diez años las tarifas se manejan de manera abierta, por medio de archivos, es decir a través de bases de datos.

En torno a las posibilidades de los empresarios tradicionales de la región para incorporarse a la industria automotriz, el empresario considera que:

[...] siempre que se trata de dinero prevalece un criterio muy importante y es el miedo... me refiero al miedo natural de un inversionista. Uno hace una apuesta a que va a tener un buen retorno sobre ella, y muchas veces uno puede tener un abanico de opciones para poner su dinero, y entonces te vas por donde hay mayor rentabilidad con el menor riesgo. Algo que pienso que pesa mucho es que los capitales tradicionales, o la gente que toma decisiones sobre los capitales tradicionales, no entienden muy bien cómo funciona esto. Ahí es donde pienso que va a jugar un papel muy importante el capital de riesgo, gente que tiene capital pero no le entiende muy bien al negocio, y hay que buscar a la persona que sí le entienda y que tenga un proyecto interesante y entonces con él coinvertir. Creo que ésta es la alternativa.

Los casos examinados reflejan algunos de los rasgos más característicos del empresariado sonorense, cuya trayectoria está fuertemente influida por su pasado agrícola y agroindustrial, y en cuya modernización las manufacturas y actividades intensivas en conocimiento han sido más bien marginales. No obstante su fuerte arraigo en la sociedad regional y su profundo conocimiento del medio, estos empresarios han desarrollado vínculos muy pobres con las actividades que en la actualidad se insertan en las cadenas globales de producción y constituyen los segmentos de mayor crecimiento en la economía regional. Para ubicar a los agentes que empiezan a emerger como el vínculo local con estas actividades hay que buscar fuera de los capitales tradicionales de la región. 


\section{Capitales tradicionales, empresarios modernos: los nuevos emprendedores locales}

A pesar de la escasa proclividad de los empresarios regionales a las inversiones de riesgo y su casi nula experiencia en actividades intensivas en conocimiento, al concluir el proceso de ampliación de la planta Ford de Hermosillo y el establecimiento de su nueva red de proveedores transnacionales, en 2005, un puñado de empresas locales habían logrado establecerse como proveedores permanentes de la armadora automotriz (Contreras, 2008a).

No eran, sin embargo, los capitales tradicionales de la región los que habían dado origen a estas empresas, sino, por lo general, negocios de reciente creación, con menos de diez años de antigüedad, que desarrollaron sus capacidades tecnológicas y empresariales en el medio industrial de las maquiladoras y de la propia industria automotriz, dedicadas a actividades como el desarrollo de software, procesos de automatización, diseño de dispositivos, maquinados de precisión y servicios de ingeniería, entre otras actividades. En ninguno de los casos identificados se trata de negocios vinculados con los capitales tradicionales del estado, sino de nuevos empresarios locales formados en el seno de la industria y sin conexión con las familias acaudaladas de la región.

Un rasgo común a estas nuevas empresas de base tecnológica es que lograron aprovechar la demanda generada por las empresas líderes para desarrollar un aprendizaje interactivo a partir de las necesidades de insumos, equipos y servicios especializados de las grandes empresas. El patrón común parte de la necesidad de transferir capacidades técnicas y gerenciales a los proveedores locales para que estén en condiciones de cumplir con los estándares de calidad y tiempos de entrega requeridos por la industria automotriz. Una vez que las empresas locales han logrado elevar sus capacidades, las nuevas competencias se convierten en un incentivo para que la empresa líder transfiera conocimientos y procesos más sofisticados en los proveedores locales, incluyendo aspectos como la logística, la ingeniería y el desarrollo de productos y de procesos. Al analizar las trayectorias de estas nuevas empresas locales, se pudieron identificar tres mecanismos principales 
de incorporación en la cadena automotriz: procesos tipo spin off; las redes socio-profesionales en el entorno regional; y la acumulación de capacidades mediante relaciones convencionales de mercado (Contreras, 2008b).

\section{Empresas creadas mediante procesos tipo spin off}

Algunas de las empresas locales más exitosas en su vinculación con la industria automotriz fueron formadas por empleados de la propia planta Ford que dejaron sus empleos para crear sus propias empresas. En su mayoría se trata de ingenieros que fueron contratados por Ford en una etapa muy temprana de sus trayectorias, y el paso por la empresa automotriz constituyó una experiencia muy relevante en la maduración de las capacidades técnicas y administrativas que más tarde pondrían al servicio de sus propios negocios.

El conocimiento adquirido por los ingenieros a través de su experiencia de trabajo en la armadora automotriz se puede clasificar en tres grandes áreas de conocimientos: técnicos, administrativos y organizacionales. El aprendizaje de carácter técnico es el que se encuentra más directamente ligado a la industria, en primer lugar a la propia industria automotriz, pero también a diferentes actividades de manufactura. En cambio el aprendizaje de carácter administrativo y organizacional es aplicable a muy diferentes actividades, sobre todo en empresas que operan con estándares internacionales.

Entre las empresas locales formadas a través de este mecanismo, se pueden mencionar a IRMI (Integración Robótica y Mantenimiento Industrial), dedicada a procesos de automatización, mantenimiento de equipos y programación, fundada en el año 2000 con diez empleados y que ahora tiene 340, y GEAR Industrial Services, con 50 empleados, fundada en 1996 y dedicada al control de calidad.

\section{Redes socio-profesionales}

El segundo mecanismo es el de las redes socio-profesionales. La operación cotidiana de las grandes empresas transnacionales da lugar a la formación de redes sociales y profesionales, formadas por el tejido 
de relaciones entre los empleados de las transnacionales y las diversas personas, instituciones y proveedores locales, a través de las cuales fluye la información y se trasmite la experiencia vinculada con el conglomerado automotriz.

Estas relaciones suelen iniciar en el ambiente universitario, por ejemplo entre estudiantes que comparten los años universitarios, y se mantienen o se reactivan una vez integrados al mercado de trabajo. Un fenómeno recurrente en las trayectorias de estas empresas es que la integración de un nuevo proveedor local surge de forma inesperada, en ocasión de alguna contingencia técnica de la gran empresa armadora o de sus proveedores de primer nivel. En condiciones de emergencia por una descompostura u otro imprevisto, la empresa líder debe acudir a los servicios de proveedores locales que puedan atender de inmediato la demanda. En estos casos las relaciones socio-profesionales son un recurso eficiente para solucionar contingencias que eventualmente derivan en relaciones más permanentes. Las transnacionales suelen encontrar así a proveedores locales confiables, y éstos a la vez descubren nichos de mercado inexplorados.

Ejemplos destacados de este tipo de empresas son Kinematics, dedicada al diseño y fabricación de manipuladores industriales, con un crecimiento de seis a 130 empleados entre 2000 y 2007, e ISPEMSA, fundada en 1996, dedicada a montaje de robots, procesos de automatización y dispositivos neumáticos e hidráulicos, que creció de ocho a 115 empleados.

\section{Relaciones de mercado}

Las empresas transnacionales buscan proveedores locales capaces de ofrecerles bajos costos, flexibilidad y calidad. Además de la pertenencia a las redes socio-profesionales y los procesos spin off, hay otros casos en los que la relación se establece fuera de las redes sociales y se origina a través de relaciones convencionales de mercado.

Tales relaciones surgen por las necesidades operativas de las grandes empresas, en este caso la Ford y sus proveedores de primero y segundo nivel. Es característico que las grandes empresas requieran de servicios generales como limpieza, vigilancia, comedores, etcétera. 
Por su propia naturaleza, este tipo de servicios suelen adquirirse en los mercados locales y con frecuencia a través de procesos competitivos, pero además se requiere de servicios más especializados como instalaciones, reparaciones de equipo, maquinados, programación, automatización, entre otros, y cuando estos servicios no son ofertados dentro de las redes socio-profesionales de la empresa, se inicia una relación con los proveedores locales a través del mercado que puede ser esporádica o bien madurar hasta estabilizarse cuando el desempeño de los proveedores locales resulta satisfactorio.

Ejemplos de este último tipo de casos son AIISA Automation, una empresa fundada en 1991, con 24 empleados actualmente y dedicada a procesos de automatización, desarrollo de software y diseño de redes, e Industrias Medina, fundada en 1986, con 48 empleados actualmente y dedicada a procesos de estampado, troquelado, punzonado, soldadura y ensambles.

\section{Conclusiones}

Las recientes inversiones automotrices en México han generado muy altas expectativas sobre las oportunidades que se presentan para las empresas locales con la expansión de esta industria. El caso del complejo industrial liderado por Ford Hermosillo muestra que estas aglomeraciones comandadas por las transnacionales generan derramas tecnológicas que propician la acumulación de capacidades y la formación de empresas locales. Procesos similares ocurren actualmente en varios estados del norte como Coahuila y Baja California, y del centro-norte como Aguascalientes y San Luis Potosí.

Lo que se encontró en esta investigación fue que la incorporación de empresas locales se ha empezado a desarrollar en los últimos años, pero esto ocurre fuera del circuito empresarial tradicional. La acumulación de capacidades tecnológicas y empresariales que permite a las empresas locales participar en la cadena automotriz involucra principalmente a empresas nuevas y se ha formado a través de su interacción directa con las empresas líderes. Ya sea a través de desprendimientos tipo spin off, de vínculos establecidos a través de las redes socio-profesionales, o de relaciones iniciadas a través del mercado, en todos los 
casos la acumulación de capacidades ocurre mediante el aprendizaje por interacción.

En general, los empresarios tradicionales de la región no han estado en condiciones de tener un papel relevante en el nuevo esquema productivo tejido alrededor de la empresa automotriz y sus grandes proveedores. El bajo perfil jugado dos décadas atrás cuando se estableció la primera planta ensambladora es un antecedente a considerar, así como el pobre desarrollo que parecen haber tenido las capacidades gerenciales de los empresarios tradicionales del estado de Sonora. Éstos se formaron y consolidaron bajo el manto protector del poder público concentrándose en actividades de bajo riesgo y ganancias rápidas. Sin embargo las entrevistas con algunos de los empresarios de Sonora parecen sugerir que la cultura gerencial de los tradicionales inversionistas regionales ha empezado a cambiar. En general mantienen sus actividades tradicionales en la agricultura, los servicios, el comercio, etc., pero también expresan un claro reconocimiento de que para crecer en un contexto competitivo es indispensable modernizarse e involucrarse, en algunos casos, en actividades manufactureras de base tecnológica. Esta línea de análisis es importante para explorar las perspectivas de los capitales regionales en el marco de las actividades emergentes de base tecnológica, sobre todo si se considera que los proveedores de la automotriz están previamente definidos mediante contratos establecidos con años de anticipación, y que las capacidades tecnológicas, organizacionales y empresariales necesarias para participar como proveedores se forman mediante un largo aprendizaje que por lo general se mide en lustros.

En el contexto de la severa crisis por la que atraviesan las grandes armadoras estadounidenses, y de la intensa competencia de éstas con las japonesas, hay razones para un moderado optimismo acerca de las posibilidades de consolidar una capa de empresas vinculadas con las cadenas globales de la industria automotriz. Pero las evidencias sugieren que el vínculo requiere de un considerable esfuerzo de apropiación por parte de las empresas locales, y los empresarios tradicionales de la región han sido en general reacios a tomar riesgos y a desplegar el esfuerzo requerido para penetrar en estos exigentes mercados. 
Por otra parte, hasta ahora la participación de las instituciones gubernamentales en este proceso ha sido tardía y poco relevante, y son los mecanismos de derrama y absorción al interior de la propia red global los que parecen haber activado la aparición de nuevas empresas locales intensivas en conocimiento. En la fase actual de la industria automotriz, caracterizada por la manufactura modular y las prácticas de subcontratación, las interacciones con el medio local se han vuelto más vastas y complejas. Esto sin duda amplía las oportunidades, pero también hace evidente la necesidad de políticas e instrumentos para aprovecharlas.

\section{Bibliografía}

Bocanegra, Carmen (2005), "Estructura y competencia entre las empresas comerciales líderes en Sonora”, Imaginales, Revista de Investigación Social, Hermosillo, Universidad de Sonora.

Carrillo Jorge y Alfredo Hualde (1998), “Third Generation Maquiladora? The Delphi-General Motors Case", Journal of Borderland Studies, vol. 13, núm. 1, pp. 79-97.

Casalet Mónica (2004), “Construcción institucional del mercado en la economía del conocimiento", Economía UNAM, núm. 2, pp. 52-63.

Casas, Rosalba y Matilde Luna (2001), “Espacios emergentes de conocimiento en las regiones: hacia una taxonomía", en Rosalba Casas (ed.), La formación de redes de conocimiento. Una perspectiva regional desde México, México, Anthropos-UnAM.

Contreras, Óscar (2000), Empresas globales, actores locales. Producción flexible y aprendizaje industrial en las maquiladoras, México, El Colegio de México.

(2008a), "Pequeñas empresas globales: aprendizaje tecnológico y creación de empresas intensivas en conocimiento en un conglomerado automotriz", Comercio Exterior (en prensa). (2008b), Empresas transnacionales, aprendizaje tecnológico y desarrollo local. Un estudio de caso en el noroeste de México. México, Instituto de Investigaciones Económicas, UNAM (en prensa). y José Guadalupe Rodríguez (2003), “Sonora en el siglo XXI: la reorganización del modelo económico", en Benjamín Burgos, 
Alejandro Mungaray y Juan Manuel Ocegueda (coordinadores), Estructura económica y demanda de educación superior en el noroeste de México, México, ANUIES-Miguel Ángel Porrúa.

Contreras, Óscar, Hirám Félix y Ulises Ponce (2000), Internet y Comercio Electrónico. ¿Una oportunidad para el desarrollo regional?, Hermosillo, El Colegio de Sonora, Serie Avances de Investigación.

Dutrénit, Gabriela, Alexandre O. Vera-Cruz, Aryenis Arias, José Luis Sanpedro y Alma Urióstegui (2006), Acumulación de capacidades tecnológicas en subsidiarias de empresas globales en México. El caso de la industria maquiladora de exportación, México, Universidad Autónoma Metropolitana-Miguel Ángel Porrúa.

Ernst Dieter (2000), Inter-Organizational Knowledge Outsourcing: What Permits Small Taiwanese Firms to Compete in the Computer Industry?, Honolulu, East-West Center, East-West Center Working Papers, Economics Series, núm. 3.

y Linsu Kim (2002), “Global Production Networks, Knowledge Diffusion, and Local Capability Formation", Research Policy, núm. 31, pp. 1417-1429.

Gereffi, Gary (1999), “International Trade and Industrial Upgrading in the Apparel Commodity Chain", Journal of International Economics, núm. 48, pp. 37-70.

Juárez, Huberto, Arturo Lara y Carmen Bueno (coord.) (2005), El auto global: cooperación y competencia en la industria au tomotriz, México, UAM, Ibero, BUAP, Conacyt.

Lara, Arturo (1998), Aprendizaje tecnológico y mercado de trabajo en las maquiladoras japonesas, México, Miguel Ángel Porrúa-UAMXochimilco.

Méndez, Rosana y Álvaro Bracamonte (2005), La pesca y la acuacultura en Sonora. Situación actual y perspectivas, Hermosillo, El Colegio de Sonora (inédito).

Moloman, Elena (2006), Modelo de exportación, desarrollo de capacidades y surgimiento de un nuevo tipo de instituciones. Comparación de Tijuana y Ciudad Juárez, tesis de maestría en Desarrollo Regional, Tijuana, El Colegio de la Frontera Norte.

Ramírez, José Carlos (1988), La nueva industrialización en Sonora: el caso de los sectores de alta tecnología, Hermosillo, El Colegio de Sonora. 
Schmitz, Hubert (2004), "Globalized Localities: Introduction", en Hubert Schmitz (ed.), Local Enterprises in The Global Economy Issues of Governance and Upgrading, Cheltenham, Reino Unido, Edward Elgar.

Solleiro, José Luis (2006), “Introducción”, en José Luis Solleiro (ed.), El sistema nacional de innovación y la competitividad del sector manufacturero en México, México, Universidad Nacional Autónoma de México.

Valenzuela, Erasmo, Jesús Robles y Álvaro Bracamonte (2005), Análisis de la competitividad en siete sistemas producto de la agricultura del noroeste de México, México, El Colegio de Sonora (inédito).

Vázquez Ruiz, Miguel Ángel (1991), Economía sonorense más allá de los valles, Hermosillo, Universidad de Sonora. y María del Carmen Hernández Moreno (2001), “Región y grupos económicos en Sonora. Breve itinerario de su integración (1910-1950)", Frontera Norte, núm. 26, vol. 13, julio-diciembre. y Guadalupe García (1992), Modernización industrial de Sonora, Hermosillo, Gobierno del Estado de Sonora, Secretaría de Fomento Educativo y Cultura.

(s/f), Integración regional en el norte de México y grupos empresariales, México, Universidad de Sonora (inédito).

Villavicencio, Daniel (2006), “El surgimiento de un entorno institucional de apoyo a las empresas maquiladoras en la frontera norte de México", en Daniel Villavicencio (ed.), La emergencia de dinámicas institucionales de apoyo a la industria maquiladora de México, México, Universidad Autónoma Metropolitana-Miguel Ángel Porrúa.

Wong, Pablo (1993), "La nueva industrialización rural en Sonora: de la villa a la fábrica global”, en Miguel Ángel Vázquez (coord.), Sonora hacia el 2000. Tendencias y desafios, Hermosillo, SINO. (1996), “La reestructuración secto-espacial en Sonora: una tipología regional", en Miguel Vázquez R. (coord.), Las regiones ante la globalización, Hermosillo, Gobierno del Estado de Sonora.

Recibido en mayo de 2008 Aprobado en septiembre de 2008 